\title{
ON MEANS WITH COUNTABLY ADDITIVE CONTINUITIES
}

\author{
LESTER E. DUBINS AND DAVID HEATH ${ }^{1}$
}

\begin{abstract}
Some notions of countable additivity, meaningful for an expectation whose domain is an arbitrary linear space of bounded, real-valued functions, are studied.
\end{abstract}

For linear spaces or ordered linear spaces which do not possess the structure of a vector lattice-important in the development of the Lebesgue-Daniell integralthere is to our knowledge no study of continuous or countably additive linear functionals other than the seminal contributions of de Finetti $[\mathbf{1}$ and 2]. The present paper was inspired mainly by de Finetti's writings.

For simplicity, linear subspaces $L$ of $l_{\infty}(\Omega)$, the space of bounded $\mathbf{R}$-valued (realvalued) functions defined on a nonempty set $\Omega$, will be considered and, on $L$, only linear functions $Q$, necessarily nonnegative, which satisfy $f \in L$ and $a \leq f \leq b$ everywhere imply $a \leq Q f \leq b$. Call such a functional a prevision, a term borrowed from de Finetti [1 and 2].

With the help of the Hahn-Banach extension theorem, it is simple to verify that each prevision on $L$ is the restriction to $L$ of a prevision $P$ on $l_{\infty}$.

Let $\Lambda_{P}$ be the collection of all $L$ such that $P$ restricted to $L$ is continuous or countably additive. In [1, Chapter 5.34 and 2, Vol. 2, Appendix 18.3], de Finetti introduces $\Lambda_{P}$ and initiates the study of its structure. He observes $L^{\prime} \subset L \in \Lambda_{p}$ implies $L^{\prime} \in \Lambda_{p}$, and he goes on to state "If $L_{1}$ and $L_{2}$ belong (to $\Lambda_{P}$ ), then so does $L_{1}+L_{2}$ (the linear space of sums $X_{1}+X_{2}, X_{1} \in L_{1}$ and $X_{2} \in L_{2}$ )."

The present paper developed in large part from the observation that the quoted assertion is erroneous. Several counterexamples, which also illustrate other phenomena, are offered. For the first, $[0,1]$ designates the closed unit interval, $C=C[0,1]$ the space of all continuous $\mathbf{R}$-valued functions with $[0,1]$ as their domain, and $\lambda$ is the usual Lebesgue integral. The indicator of a set is, as usual, the function that assumes the value 1 on the set 0 off the set. The useful convention introduced by de Finetti of designating a set and its indicator by the same letter will usually be adopted here.

EXAMPLE 1. Let $\varphi$ be an open dense subset of $[0,1]$ with $\lambda \varphi<1, L_{1}=$ $C[0,1], L_{2}=\{t \varphi: t \in R\}, L=L_{1}+L_{2}$ and $P(\theta+t \varphi)=\lambda \theta+t, \theta \in C[0,1]$.

To formulate the strong sense in which Example 1 is a counterexample, it is necessary to articulate two definitions. If $P f_{n} \rightarrow 0$ for every decreasing sequence $f_{n} \in L$ that converges pointwise to 0 , call $P m$-continuous on $L$ ( $m$ for 'monotone'). Let $\sigma(L)$ designate the smallest sigma field of subsets of $\Omega$ such that $f^{-1}(B) \in \sigma(L)$

Received by the editors February 21, 1983 and, in revised form, August 29, 1983.

1980 Mathematics Subject Classification. Primary 28C05, 46G12, 60A05.

Key words and phrases. Probability, prevision, linear forms, countable additivity, finite additivity, expectation.

${ }^{1}$ Research sponsored by NSF Grant MCS-80-02535 
for all $f \in L$ and all Borel subsets $B$ of $\mathbf{R}$. Call $P$ countably additive on $L$ if, for some probability $Q$ which is countably additive on $\sigma(L), P f$ is $\int f d Q$ for each $f \in L$. In Example 1, $P$ is countably additive on both $L_{1}$ and $L_{2}$, but on their join, $L_{1}+L_{2}, P$ is not even $m$-continuous.

A second example, offered by David Gilat and presented here with his permission, shows that on the join of two spaces on each of which $P$ is countably additive, $P$ can be purely finitely additive, which means that every extension of $P$ to $\sigma(L)$ assigns probability 1 to the union of a countable collection of events each of which has $P$-probability zero.

Let $Z_{+}$be the set of nonnegative integers, $Z_{-}$the set of negative integers, and

$$
Z=Z_{+} \cup Z_{-} \text {. }
$$

EXAMPLE 2 (GILAT). $L$ is the set of $f \in l_{\infty}(Z)$ such that $f$ differs from a constant on at most a finite subset of $Z, P(f)$ is the constant corresponding to $f, L_{-}$is the set of $f \in L$ such that $f$ is constant on $Z_{-}$, and $L_{+}$is the set of $f \in L$ such that $f$ is constant on $Z_{+}$.

A notion of continuity stronger than $m$-continuity can be introduced. If $P f_{n} \rightarrow 0$ for every uniformly bounded sequence $f_{n} \in L$ which converges to 0 pointwise, $P$ is $d$-continuous (' $d$ ' for 'dominated'). That countable additivity implies $d$-continuity and that $d$-continuity implies $m$-continuity are trivialities. That the first implication cannot be reversed is an immediate corollary to

Proposition 1. There is a $P$ and an $L$ such that on $L, P$ is d-continuous but on the $P$-completion of $L, P$ fails to be d-continuous. Indeed, for every purely finitely additive probability $P$ on $l_{\infty}(Z)$, there is an $L \subset l_{\infty}(Z)$ on which $P$ is $d$-continuous but whose $P$-completion is $l_{\infty}(Z)$.

ProOF. Fix a purely finitely additive $P$ on $l_{\infty}(Z)$ and let $L$ be the set of bounded $f$ such that $P f$ equals $2 f(2)-f(1)$. On $L, P$ is plainly $d$-continuous. What remains to be seen is that for every bounded $f$ there is a $g \in L$ and an $h \in L$ such that $g \leq f \leq h$ and $P h-P g$ is arbitrarily small. To this end, let $c$ be a real number and let $f_{c}$ agree with $f$ everywhere except that $f_{c}(2)=c$ and $f_{c}(1)$ is so chosen that $f_{c} \in L$, that is, $P f_{c}=2 f_{c}(2)-f_{c}(1)$ or, equivalently,

$$
P f_{c}=2 c-f_{c}(1) \text {. }
$$

Plainly, $P f_{c}=P f$; for large $c, f_{c} \geq f$; and for small $c, f_{c} \leq f$. So $f$ belongs to the completion of the restriction of $P$ to $L$.

COROllary 1. There is a $P$ and an $L$ such that on $L, P$ is d-continuous but not countably additive.

Here is an example which shows that $m$-continuity does not imply $d$-continuity.

EXAMPLE 3 . Let $\Omega$ and $\Omega^{\prime}$ be disjoint infinite sets, $E_{1}, E_{2}, \ldots$ a logically independent sequence of subsets of $\Omega, B_{1} \supset B_{2} \supset \cdots$ a strictly decreasing sequence of subsets of $\Omega^{\prime}$ whose intersection is empty, $d_{n}>0$ with $\sum d_{n}<\infty, f_{n}=$ $B_{n}+d_{n}\left(2 E_{n}-1\right), L$ the linear space generated by $f_{1}, f_{2}, \ldots$, and $P f_{n}=1$.

The notion of logically independent random numbers, $X_{1}, \ldots, X_{n}$, is formulated in de Finetti [2, Vol. 1]. In the special case that each $X_{i}$ has precisely two possible values, this simply means that the $n$-tuple $X_{1}, \ldots, X_{n}$ has $2^{n}$ possible values. 
The following are useful preliminaries to the proof that the $P$ of Example 3 is indeed $m$-continuous but not $d$-continuous on $L$.

Stochastically independent events are necessarily logically independent. For an example of interest, let $E_{i}$ be the set of positive integral multiples of the $i$ th prime. In the following lemma, events are again identified with their indicators.

Lemma 1. Suppose $E_{1}, E_{2}, \ldots$ are logically independent sets and let $X_{i}$ designate $2 E_{i}-1$. Then the only finite linear combination of the $X_{i}$ which is everywhere nonnegative is identically zero. Moreover, the $X_{i}$ are logically, and, a fortiori, linearly independent. Therefore, if $f=\sum c_{i} X_{i}(1 \leq i \leq n)$ is everywhere nonnegative, every $c_{i}$ is 0 and, therefore, on the linear span of $X_{1}, X_{2}, \ldots$, every $P$ is m-continuous.

The lemma has been formulated as a sequence of assertions which, if verified in order, makes its proof straightforward.

That $P$ in Example 3 is $m$-continuous on $L$ is easily verified with the help of Lemma 1 . To see that $P$ fails to be $d$-continuous on $L$, verify that $f_{n}$ is uniformly bounded and converges to zero, but $P f_{n}=1$ for all $n$.

For another phenomenon, let $L+1$ designate the linear span of $L$ and the constant function 1 .

Proposition 2. There is a $P$ and an $L-$ which is norm-complete-such that, on $L, P$ is d-continuous but on $L+1, P$ is not even $m$-continuous. Moreover on $L+1, P$ is purely finitely additive.

PRELIMINARIES TO THE PROOF OF PROPOSITION 2. A sweep of a topological space $X$ is a sequence of continuous, real-valued functions $f_{1}, f_{2}, \ldots$ with domain $X$ and with values in the closed unit interval such that (a) for each $n$, there is a compact set $K_{n}$ on whose complement $f_{n}$ assumes only the value 1 and (b) for each $x \in X$ there is an $f_{n}$ that vanishes at $x$.

Use the usual notation $C(X)$ for the set of continuous $R$-valued functions, $f$, with domain $X$ that converge to a limit at infinity and let $P_{X} f$ be that limit.

LEMMA 2. Each of the following conditions implies its successor.

(i) $X$ is a noncompact, locally compact, sigma compact topological space.

(ii) $X$ possesses a sweep.

(iii) $X$ possesses a nonincreasing sweep.

(iv) On $C(X), P_{X}$ is not $m$-continuous and is, in fact, purely finitely additive.

PROOF. (i) $\rightarrow$ (ii) is easy in view of the well-known result of Urysohn that if a pair of disjoint closed subsets of a topological space can be separated by open sets then they can even be separated by a real-valued continuous function.

(ii) $\rightarrow$ (iii) is trivial.

(iii) $\rightarrow$ (iv). Let $\left\{f_{n}\right\}$ be a nonincreasing sweep of $X$. Then $P_{X} f_{n}=1$ for all $n$ and $f_{n} \searrow 0$, so $P_{X}$ is not $m$-continuous. Let $L_{n}(x)$ be 1 or 0 according as $f_{n}(x)=0$ or $f_{n}(x)>0$. Plainly, $0 \leq L_{n} \leq 1-f_{n}$. Therefore,

$$
0 \leq P L_{n} \leq 1-P f_{n}=1-P X f_{n}=1-1=0
$$

for any prevision $P$ on $l_{\infty}(X)$ that extends $P_{X}$. So $P\left(L_{n}\right)=0$, that is,

$$
P\left(f_{n}=0\right)=0 \text { for all } n \text {. }
$$


Since $X$ is the union of the events $\left(f_{n}=0\right), P$ is purely finitely additive.

Incidentally, whenever $X$ satisfies the conditions of Lemma 2(i), each prevision on $C(X)$ is uniquely expressible as a convex combination of $P_{X}$ with a prevision that is countably additive on $C(X)$. This implies, of course, that there is no purely finitely additive prevision on $C(X)$ other than $P_{X}$.

Proof of Proposition 2 CONTINUEd. Plainly, the restriction of $P_{X}$ to $C_{0}(X)$, that is, to those $f \in C(X)$ for which $P_{X} f=0$, is $d$-continuous. Since $C(X)$ is plainly the linear span of $C_{0}(X)$ with the constant function 1, Lemma 2 applies with $L=C_{0}(X)$ and $P=P_{X}$.

The question arises, for the various forms of continuity, whether continuity of a $P$ on an $L$ implies its continuity on the uniform closure, $L^{u}$, of $L$. For $d$-continuity the answer is affirmative according to the next proposition which, though quite simple, contrasts with Propositions 1 and 2.

Proposition 3. If $P$ is $d$-continuous on $L$, then $P$ is $d$-continuous on the uniform closure of $L$.

Proof. Let $a \leq g_{n} \leq b, g_{n} \in L^{u}, g_{n} \rightarrow 0$ pointwise. Then, there exists $f_{n} \in L,\left\|f_{n}-g_{n}\right\| \rightarrow 0$ as $n \rightarrow \infty$. Plainly, $f_{n}$ is uniformly bounded and $f_{n} \rightarrow 0$ pointwise. Consequently, $P f_{n} \rightarrow 0$. Moreover,

$$
\left|P g_{n}-P f_{n}\right|=\left|P\left(g_{n}-f_{n}\right)\right| \leq\left\|f_{n}-g_{n}\right\|, \quad \text { so } P g_{n} \rightarrow 0 .
$$

(Perhaps some reader will have an interest to settle whether $m$-continuity on $L$ implies $m$-continuity on its uniform closure.)

Stimulated by a conversation with Lucien Le Cam, various questions arise of this nature: given $L \subset L^{\prime} \subset L^{\prime \prime}$ and a $P$ on $L$ that has only one countably additive or continuous extension to $L^{\prime \prime}$, can it possess more than one countably additive or continuous extension to $L^{\prime}$ ? Here, only a simple, and no doubt well-known, example is recorded to show that $P$ on $L$ could have a unique countably additive extension to $L^{\prime \prime}$ but several $d$-continuous extensions to $L^{\prime}$.

EXAMPLE 4. $L^{\prime \prime}$ is the set of bounded Borel functions defined on the closed unit interval, $L \subset L^{\prime \prime}$ consists of those $f$ that are everywhere continuous, $L^{\prime}$ is the linear span of $L$ and the indicator of the irrationals, and $P$ on $L$ is the Lebesgue integral.

PROOF FOR EXAMPLE 4. It is only necessary to verify that $P$ admits of more than one $d$-continuous extension to $L^{\prime}$. To this end, let $P$ assign any probability to the irrationals. That such a $P$ is $d$-continuous is easily verified, for the indicator of the irrationals is not the pointwise limit of a sequence of continuous functions, in view of a theorem of Baire which asserts that any such limit possesses points of continuity.

Since $m$-continuity, $d$-continuity and countable additivity are distinct notions, and since de Finetti (in the references cited above) provides still another interesting variant of the notion of continuity, it follows that, for $\Lambda_{p}$ as defined above, to designate a well-specified collection of $L$, the notion of continuity must, of course, first be specified.

ACKNOWLEDGEMENT. Our thanks go to the referee whose suggestions led to improvements in this note. 


\section{REFERENCES}

1. Bruno de Finetti, Probability, induction, and statistics, Wiley, New York, 1972.

2. _ , Theory of probability, Vols. 1 and 2, Wiley, New York, 1975.

Department of Mathematics, University of California, Berkeley, CaliforNIA 94720

DEPARTMENT OF OPERATIONS RESEARCH AND INDUSTRIAL ENGINEERING, CORNELL UNIVERSITY, ITHACA, NEW YORK 14853 